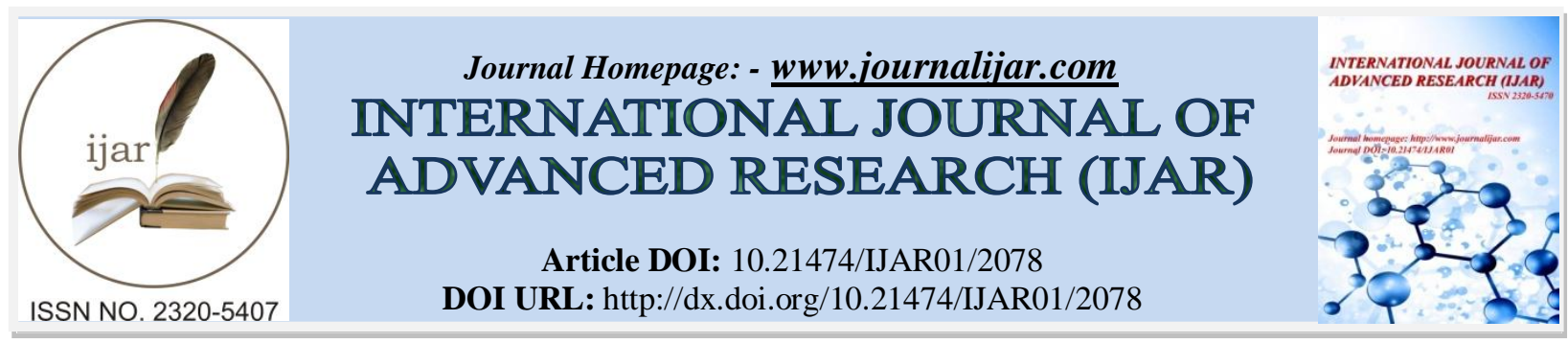

RESEARCH ARTICLE

\title{
EFFECT OF PACKAGING MATERIALS AND STORAGE CONDITIONS ON SHELF LIFE AND QUALITY OF OKRA*
}

\author{
H. D. Indore, V. K. Garande ${ }^{1}$, S. S. Dhumal ${ }^{2}$, D. R. Patgaonkar ${ }^{2}$, V. S. Patil ${ }^{3}$ and P. N. Sonawane ${ }^{4}$. \\ 1. Professor of Horticulture, College of Agriculture, Kolhapur - 416004 (MPKV, Rahuri) \\ 2. Asstt. Prof. of Horticulture, College of Agriculture, Kolhapur - 416004 (MPKV, Rahuri) \\ 3. Asstt. Prof. of Plant Pathology \& Micro-biology, College of Agriculture, Kolhapur - 416004 (MPKV, Rahuri) \\ 4. Jr. Research Assistant, Department of Horticulture, College of Agriculture, Kolhapur - 416004 (MPKV, Rahuri)
}

Manuscript Information:

\section{Manuscript History}

Received: 24 September 2016

Final Accepted: 26 October 2016

Published: November 2016

Key words:-

Okra, packaging material, storage

conditions, shelf life, quality.

\section{Abstract}

The present study was undertaken with consisting of three storage conditions (room temperature, zero energy cool chamber and refrigerated storage) as main factor and six types of packaging material such as 150, 200, 300, 400 gauge polyethylene bags with $2 \%$ vents, punnets and control i.e. without packaging with three replications in factorial randomized block design during summer season of 2015. The physiological loss in weight exhibited increasing trend while the fruit firmness, total chlorophyll and ascorbic acid content and sensorial qualities of okra fruits exhibited decreasing trend through the storage period irrespective packaging materials and storage conditions. The shelf life of okra was found to be three days at room temperature, nine days at zero energy cool chamber and fifteen days at refrigerated storage when packed in 400 gauge polyethylene bags with $2 \%$ vents with more retention of organoleptic qualities and minimum total microbial counts.

Copy Right, IJAR, 2016,. All rights reserved.

\section{Introduction:-}

Okra (Abelmoschus esculentus (L.), Moench) also known as Lady's finger is an important vegetable crop and belongs to family Malvaceae. It is major vegetable being cultivated throughout India particularly in states of Uttar Pradesh, Bihar, Orissa, West Bengal, Andhra Pradesh, Karnataka, Assam, Maharashtra and Gujarat (Anon., 2014). The Okra has prominent position among the vegetables due to its wide adaptability, year round cultivation, export potential and high nutritive value as well as medicinal value. It is good source of vitamin A, B and C and also rich source of proteins and minerals. It is also excellent source of iodine and is useful for the treatment of goiter. It is also good source of fibre hence it is used in prevention of many diseases and disorders (Gopalkrishnan, 2007). Fresh vegetables are inherently perishable, during the process of distribution and marketing a substantial losses are incurred which ranges from a slight loss of quality to total spoilage which can be avoided by giving proper prestorage treatments such as pre-cooling, packaging, low temperature storage etc. Resistance to water vapour permeability and freedom from undesirable flavour is an important consideration in the choice of suitable packaging materials (Leonard, 1987).

* A part of thesis submitted by the senior author for the award M. Sc (Hort.) degree.

Corresponding Author:- Dr. V. K. Garande.

Address:- Professor of Horticulture College of Agriculture, Kolhapur - 416004 (MPKV Rahuri) 
Major factors affecting storage of fruits and vegetables are environmental factors viz; temperature, relative humidity, composition and proportion of gases in storage and exposure to light. At high temperature okra exhibits high rate of respiration resulting in rapid production of heat and subsequent deterioration. Therefore, it is necessary to find out the suitable packaging material and method of storage for okra during summer months. Hence, the present investigation was undertaken at College of Agriculture, Kolhapur to study the effect of packaging materials and storage conditions on storage behaviour of okra.

\section{Materials and methods:- Sample preparation:-}

The tender, healthy and freshly harvested okra fruits were brought in Postharvest Technology Laboratory of Horticulture Section, College of Agriculture, Kolhapur and were sorted out manually in order to remove damaged, bruised, misshaped over aged and diseased fruits. About $250 \mathrm{~g}$ of okra fruits were packed in different packaging material under study and stored at ambient temperature, zero energy cool chamber (90-95\% RH) and refrigerated storage (RS) $10-13^{\circ} \mathrm{C}$ and $85-90 \% \mathrm{RH}$.

\section{Bio-chemical analysis:-}

Physiological loss in weight of okra was determined by weighing the samples before and after each storage interval and expressed in terms of percentage. The fruit firmness of the okra was measured with the help of firmness tester in terms of $\mathrm{kgcm}^{-2}$. The ascorbic acid content was estimated by 2, 6-dichlorophenol indophenols-dye and the total chlorophyll content was extracted in $80 \%$ acetone and the absorbance at $663 \mathrm{~nm}$ and $645 \mathrm{~nm}$ was read on spectrophotometer and expressed in terms of $\operatorname{mg}_{100 \mathrm{~g}^{-1}}(\mathrm{AOAC}, 1990)$.

\section{Microbial Analysis:-}

Total yeast and mould count of okra was determined by following the standard procedures described in USFDA (BAM) IS 5401: 2002, 5402: 2002 (Anon, 2011).

\section{Sensorial analysis:-}

The sensory evaluation was performed by a panel of seven trained judges for colour, tenderness, appearance and overall acceptability of okra by Amerine, et al. (1965) on nine point hedonic scale.

\section{Statistical analysis:-}

The data with respect to physico-chemical and sensory parameters was analyzed for the statistical significance according to the procedure given by Panse and Sukhatme (1985).

\section{Results and Discussion:- \\ Bio-chemical composition of okra:- \\ Physiological loss in weight (PLW, \%):-}

The physiological loss in weight of okra was significantly increased with increase in storage period in all 18 treatment combinations as shown in Table 1 . At the end of the storage period at ambient temperature i.e. after 3 day of storage, $\mathrm{S}_{1} \mathrm{~T}_{4}$ (400 gauge polyethylene bag with $2 \%$ vents) recorded the lowest physiological loss in weight $(3.50 \%)$ followed by treatment combination $S_{1} T_{2}$ and $S_{1} T_{3}(3.61 \%)$, whereas the highest physiological loss in weight was recorded in the treatment combination $\mathrm{S}_{1} \mathrm{~T}_{5}(3.83 \%)$. The similar trend was observed in case of zero energy cool chamber, the treatment $S_{2} T_{4}$ recorded the lowest physiological loss in weight $(6.57 \%)$ closely followed by $S_{2} T_{3}$ $(6.60 \%)$ at the end of ninth day storage. Under refrigerated storage conditions, the treatment $\mathrm{S}_{3} \mathrm{~T}_{4}$ i.e. 400 gauge polyethylene with $2 \%$ vents registered the minimum physiological loss in weight of $7.50 \%$ closely followed by $\mathrm{S}_{3} \mathrm{~T}_{3}$ $(7.57 \%)$ while the highest physiological loss in weight was registered in $\mathrm{S}_{3} \mathrm{~T}_{5}(8.11 \%)$ at the end of $15^{\text {th }}$ day of storage. The weight loss was due to uncontrolled water loss and food reserve from tissues of okra due to biochemical activities such as transpiration and respiration (Santi, et al., 1992). The results of present investigation are in close conformity with results reported by Roy and Khurdiya (1986), Negi and Roy (2004), Koraddi (2005) and Reddy, et al. (2013) in different vegetables.

Fruit firmness $\left(\mathrm{kgcm}^{-2}\right)$ :-

The firmness of okra significantly influenced by different packaging materials and storage conditions (Table 1). It was observed that the firmness was found to be decreased rapidly during storage conditions. The rate of decrease was found to be faster under room temperature as compared to zero energy cool chamber and refrigerated storage. The firmness of okra samples packed in 400 gauge polyethylene bags with $2 \%$ vent and stored at refrigerated 
storage was found to be $5.13 \mathrm{kgcm}^{-2}$ closely followed by the treatment $T_{3},\left(5.12 \mathrm{kgcm}^{-2)}\right.$ at the end of $15^{\text {th }}$ day of storage while minimum firmness was recorded in $\mathrm{S}_{3} \mathrm{~T}_{5}\left(5.09 \mathrm{kgcm}^{-2}\right)$. At the end of storage period, the treatment $\mathrm{S}_{2} \mathrm{~T}_{4}$ (400 gauge polyethylene with $2 \%$ vent) registered the highest firmness $\left(5.18 \mathrm{kgcm}^{-2}\right)$ immediately followed by $\mathrm{S}_{2} \mathrm{~T}_{3}\left(5.17 \mathrm{kgcm}^{-2}\right)$ on $9^{\text {th }}$ day of storage under cool chamber. The decrease in firmness of okra might be due to loss in moisture during storage. These results are in accordance with the results reported by Jain, et al. (2014) in okra.

Table 1:- Effect of packaging materials and storage conditions on physiological loss in weight (\%) and fruit firmness

\begin{tabular}{|c|c|c|c|c|c|c|}
\hline & \multicolumn{3}{|c|}{ Physiological Loss in Weight } & \multicolumn{3}{|c|}{ Fruit Firmness（Initial: 5.29) } \\
\hline & \multicolumn{6}{|c|}{ Storage period (days) } \\
\hline Storage & 3 & 9 & 15 & 3 & 9 & 15 \\
\hline $\mathrm{S}_{1}$ & 3.67 & - & - & 5.23 & - & - \\
\hline $\mathrm{S}_{2}$ & 2.22 & 6.64 & - & 5.24 & 5.16 & - \\
\hline $\mathrm{S}_{3}$ & 1.31 & 5.34 & 7.69 & 5.26 & 5.22 & 5.11 \\
\hline SE $(\mathbf{m}) \pm$ & 0.03 & 0.03 & - & 0.00 & 0.01 & - \\
\hline CD at $1 \%$ & 0.11 & 0.13 & - & 0.01 & 0.02 & - \\
\hline \multicolumn{7}{|l|}{ Treatments } \\
\hline $\mathrm{T}_{1}$ & 2.04 & 6.06 & 7.67 & 5.24 & 5.18 & 5.10 \\
\hline $\mathrm{T}_{2}$ & 1.96 & 5.99 & 7.61 & 5.25 & 5.19 & 5.11 \\
\hline $\mathrm{T}_{3}$ & 1.91 & 5.93 & 7.57 & 5.25 & 5.20 & 5.12 \\
\hline $\mathrm{T}_{4}$ & 1.83 & 5.89 & 7.50 & 5.26 & 5.21 & 5.13 \\
\hline $\mathrm{T}_{5}$ & 2.08 & 6.08 & 8.11 & 5.23 & 5.17 & 5.09 \\
\hline $\mathrm{T}_{6}$ & 7.83 & 0.00 & 0.00 & 5.22 & 0.00 & 0.00 \\
\hline $\mathrm{SE}(\mathrm{m}) \pm$ & 0.04 & 0.06 & - & 0.00 & 0.01 & - \\
\hline CD at $1 \%$ & 0.15 & 0.23 & - & 0.01 & 0.04 & - \\
\hline \multicolumn{7}{|l|}{ Interaction } \\
\hline $\mathrm{S}_{1} \mathrm{~T}_{1}$ & 3.78 & - & - & 5.22 & - & - \\
\hline$S_{1} T_{2}$ & 3.61 & - & - & 5.24 & - & - \\
\hline $\mathrm{S}_{1} \mathrm{~T}_{3}$ & 3.61 & - & - & 5.24 & - & - \\
\hline $\mathrm{S}_{1} \mathrm{~T}_{4}$ & 3.50 & - & - & 5.25 & - & - \\
\hline $\mathrm{S}_{1} \mathrm{~T}_{5}$ & 3.83 & - & - & 5.21 & - & - \\
\hline $\mathrm{S}_{1} \mathrm{~T}_{6}$ & - & - & - & - & - & - \\
\hline $\mathrm{S}_{2} \mathrm{~T}_{1}$ & 2.33 & 6.72 & - & 5.24 & 5.15 & - \\
\hline $\mathrm{S}_{2} \mathrm{~T}_{2}$ & 2.28 & 6.64 & - & 5.24 & 5.16 & - \\
\hline $\mathrm{S}_{2} \mathrm{~T}_{3}$ & 2.11 & 6.60 & - & 5.25 & 5.17 & - \\
\hline $\mathrm{S}_{2} \mathrm{~T}_{4}$ & 2.00 & 6.57 & - & 5.25 & 5.18 & - \\
\hline $\mathrm{S}_{2} \mathrm{~T}_{5}$ & 2.40 & 6.67 & - & 5.23 & 5.14 & - \\
\hline $\mathrm{S}_{2} \mathrm{~T}_{6}$ & - & & - & - & - & - \\
\hline $\mathrm{S}_{3} \mathrm{~T}_{1}$ & 0.00 & 5.40 & 7.67 & 5.26 & 5.21 & 5.10 \\
\hline $\mathrm{S}_{3} \mathrm{~T}_{2}$ & 0.00 & 5.33 & 7.61 & 5.27 & 5.22 & 5.11 \\
\hline $\mathrm{S}_{3} \mathrm{~T}_{3}$ & 0.00 & 5.25 & 7.57 & 5.27 & 5.23 & 5.12 \\
\hline $\mathrm{S}_{3} \mathrm{~T}_{4}$ & 0.00 & 5.20 & 7.50 & 5.27 & 5.24 & 5.13 \\
\hline $\mathrm{S}_{3} \mathrm{~T}_{5}$ & 0.00 & 5.50 & 8.11 & 5.26 & 5.20 & 5.09 \\
\hline $\mathrm{S}_{3} \mathrm{~T}_{6}$ & 7.83 & - & - & 5.22 & - & - \\
\hline SE (m) \pm & 0.07 & 0.08 & 0.13 & 0.01 & 0.01 & 0.01 \\
\hline CD at $1 \%$ & 0.27 & 0.33 & 0.60 & 0.02 & 0.05 & 0.05 \\
\hline \multicolumn{7}{|c|}{$\mathrm{T}_{1}-150$ gauge polyethylene with $2 \%$ vent } \\
\hline \multicolumn{2}{|c|}{$\mathrm{S}_{1}$-Ambient temperature } & \multicolumn{5}{|c|}{$\mathrm{T}_{2}-200$ gauge polyethylene with $2 \%$ vent } \\
\hline \multicolumn{2}{|c|}{$\mathrm{S}_{2}$-Zero Energy Cool Chamber } & \multicolumn{5}{|c|}{$\mathrm{T}_{3}-300$ gauge polyethylene with $2 \%$ vent } \\
\hline \multirow{3}{*}{\multicolumn{2}{|c|}{$\mathrm{S}_{3}$-Refrigerated storage }} & \multicolumn{5}{|c|}{$\mathrm{T}_{4}-400$ gauge polyethylene with $2 \%$ vent } \\
\hline & & \multicolumn{5}{|c|}{$\mathrm{T}_{5}$ - Punnet } \\
\hline & & \multicolumn{5}{|c|}{$\mathrm{T}_{6}-$ Control } \\
\hline
\end{tabular}

Total chlorophyll content $\left(\mathrm{mg} 100 \mathrm{~g}^{-1}\right)$ :-

The total chlorophyll content of okra was significantly influenced by different packaging material and storage conditions (Table 2). The maximum total chlorophyll content of okra was recorded in refrigerated storage (8.62 $\left.\mathrm{mg} 100 \mathrm{~g}^{-1}\right)$ followed by cool chamber $\left(8.25 \mathrm{mg} 100 \mathrm{~g}^{-1}\right)$ and ambient temperature $\left(7.29 \mathrm{mg} 100 \mathrm{~g}^{-1}\right)$ at the end of $3^{\text {rd }}$ day 
of storage. Among the treatment combinations the highest total chlorophyll content was recorded in the samples packed in 400 gauge polyethylene bags with $2 \%$ vent $\left(4.20 \mathrm{mg} 100 \mathrm{~g}^{-1}\right)$ closely followed by $\mathrm{S}_{3} \mathrm{~T}_{3}\left(4.17 \mathrm{mg} 100 \mathrm{~g}^{-1}\right)$ at the end of $15^{\text {th }}$ day of storage under refrigerated conditions. The chlorophyll content was decreased with increase in temperature and decrease in humidity. Higher losses of chlorophyll may be due to degradation of chlorophyll which is accelerated by exposure to higher temperature and low humidity. Presence of vents has failed to increase carbon dioxide concentration, thus leading to higher amount of yellowing. The principle cause of the breakdown of chlorophyll is the change in $\mathrm{pH}$ mainly due to leakage of organic acids from the vacuole, oxidative system and chlorophyllases (Wills, et al., 1989). The decreasing trend of chlorophyll content with advancement of storage has been reported Abe and Watada, (1991) and Rai, et al. (2009) in shredded cabbage.

Table 2:- Effect of packaging materials and storage conditions on total chlorophyll and ascorbic acid contents $\left(\mathrm{mg} 100 \mathrm{~g}^{-1}\right)$ of okra during storage.

\begin{tabular}{|c|c|c|c|c|c|c|}
\hline \multirow{3}{*}{ Storage } & \multicolumn{3}{|c|}{ Total chlorophyll (Initial : 9.89) } & \multicolumn{3}{|c|}{ Ascorbic acid (Initial : 14.00) } \\
\hline & \multicolumn{6}{|c|}{ Storage period (days) } \\
\hline & 3 & 9 & 15 & 3 & 9 & 15 \\
\hline $\mathrm{S}_{1}$ & 7.29 & - & - & 10.38 & 10.43 & - \\
\hline $\mathrm{S}_{2}$ & 8.25 & 6.83 & - & 12.54 & 11.27 & - \\
\hline $\mathrm{S}_{3}$ & 8.62 & 7.60 & 4.11 & 13.35 & 0.07 & 8.72 \\
\hline SE $(\mathbf{m}) \pm$ & 0.02 & 0.00 & - & 0.02 & 0.30 & - \\
\hline CD at $1 \%$ & 0.05 & 0.01 & - & 0.07 & & - \\
\hline Treatments & & & & & 10.84 & \\
\hline $\mathrm{T}_{1}$ & 8.14 & 7.21 & 4.07 & 12.04 & 10.85 & 8.64 \\
\hline $\mathrm{T}_{2}$ & 8.15 & 7.23 & 4.10 & 12.15 & 10.87 & 8.73 \\
\hline $\mathrm{T}_{3}$ & 8.19 & 7.24 & 4.17 & 12.28 & 10.88 & 8.80 \\
\hline $\mathrm{T}_{4}$ & 8.17 & 7.24 & 4.20 & 12.36 & 10.82 & 8.85 \\
\hline$T_{5}$ & 8.01 & 7.16 & 4.00 & 11.95 & 0.00 & 8.56 \\
\hline $\mathrm{T}_{6}$ & 7.49 & 0.00 & 0.00 & 12.35 & 0.10 & 0.00 \\
\hline $\mathrm{SE}(\mathrm{m}) \pm$ & 0.03 & 0.00 & - & 0.02 & 0.43 & - \\
\hline CD at $1 \%$ & 0.08 & 0.01 & - & 0.10 & & - \\
\hline Interaction & & & & & - & \\
\hline $\mathrm{S}_{1} \mathrm{~T}_{1}$ & 7.37 & - & - & 10.25 & - & - \\
\hline $\mathrm{S}_{1} \mathrm{~T}_{2}$ & 7.37 & - & - & 10.38 & - & - \\
\hline $\mathrm{S}_{1} \mathrm{~T}_{3}$ & 7.40 & - & - & 10.54 & - & - \\
\hline $\mathrm{S}_{1} \mathrm{~T}_{4}$ & 7.33 & - & - & 10.58 & - & - \\
\hline $\mathrm{S}_{1} \mathrm{~T}_{5}$ & 7.00 & - & - & 10.13 & - & - \\
\hline $\mathrm{S}_{1} \mathrm{~T}_{6}$ & - & - & - & - & 10.41 & - \\
\hline $\mathrm{S}_{2} \mathrm{~T}_{1}$ & 8.22 & 6.81 & - & 12.43 & 10.43 & - \\
\hline $\mathrm{S}_{2} \mathrm{~T}_{2}$ & 8.25 & 6.83 & - & 12.48 & 10.46 & - \\
\hline $\mathrm{S}_{2} \mathrm{~T}_{3}$ & 8.30 & 6.84 & - & 12.66 & 10.48 & - \\
\hline $\mathrm{S}_{2} \mathrm{~T}_{4}$ & 8.30 & 6.84 & - & 12.78 & 10.38 & - \\
\hline $\mathrm{S}_{2} \mathrm{~T}_{5}$ & 8.20 & 6.80 & - & 12.35 & - & - \\
\hline $\mathrm{S}_{2} \mathrm{~T}_{6}$ & - & - & - & - & 11.26 & - \\
\hline $\mathrm{S}_{3} \mathrm{~T}_{1}$ & 8.84 & 7.60 & 4.07 & 13.43 & 11.27 & 8.64 \\
\hline $\mathrm{S}_{3} \mathrm{~T}_{2}$ & 8.85 & 7.62 & 4.10 & 13.59 & 11.28 & 8.73 \\
\hline $\mathrm{S}_{3} \mathrm{~T}_{3}$ & 8.87 & 7.63 & 4.17 & 13.66 & 11.28 & 8.80 \\
\hline $\mathrm{S}_{3} \mathrm{~T}_{4}$ & 8.87 & 7.64 & 4.20 & 13.72 & 11.26 & 8.85 \\
\hline $\mathrm{S}_{3} \mathrm{~T}_{5}$ & 8.83 & 7.52 & 4.00 & 13.35 & - & 8.56 \\
\hline $\mathrm{S}_{3} \mathrm{~T}_{6}$ & 7.49 & - & - & 12.35 & 0.17 & - \\
\hline $\mathrm{SE}(\mathrm{m}) \pm$ & 0.05 & 0.01 & 0.02 & 0.04 & 0.74 & 0.02 \\
\hline CD at 1\% & 0.13 & 0.02 & 0.06 & 0.17 & & 0.08 \\
\hline \multicolumn{3}{|c|}{ "` indicate termination } & \multicolumn{4}{|c|}{$\mathrm{T}_{1^{-}}-150$ gauge polyethylene with $2 \%$ vent } \\
\hline \multicolumn{3}{|c|}{$S_{1}$-Ambient temperature } & \multicolumn{4}{|c|}{$\mathrm{T}_{2}-200$ gauge polyethylene with $2 \%$ vent } \\
\hline \multicolumn{3}{|c|}{$\mathrm{S}_{2}$-Zero Energy Cool Chamber } & \multicolumn{4}{|c|}{$\mathrm{T}_{3}-300$ gauge polyethylene with $2 \%$ vent } \\
\hline \multirow{3}{*}{\multicolumn{3}{|c|}{$\mathrm{S}_{3}$-Refrigerated storage }} & \multicolumn{4}{|c|}{$\mathrm{T}_{4}-400$ gauge polyethylene with $2 \%$ vent } \\
\hline & & & \multicolumn{4}{|c|}{$\mathrm{T}_{5}$ - Punnet } \\
\hline & & & \multicolumn{4}{|c|}{$\mathrm{T}_{6^{-}}$Control } \\
\hline
\end{tabular}


Ascorbic acid content $\left(\mathrm{mg}^{100 \mathrm{~g}^{-1}}\right)$ :-

The ascorbic acid content of okra fruit was found to be decreased significantly with increase in storage period irrespective of packaging materials and storage conditions (Table 2). However, the rate of decrease was found to be faster under room temperature as compared to zero energy cool chamber and refrigerated storage. At the end of ninth day of storage under cool chamber, the highest ascorbic acid content was noticed in the treatment $\mathrm{S}_{2} \mathrm{~T}_{3}(10.48$ $\mathrm{mg} 100 \mathrm{~g}^{-1}$ ) which was at par with the remaining treatments. The treatment combination $\mathrm{S}_{3} \mathrm{~T}_{4}$ (400 gauge polyethylene with $2 \%$ vent) recorded the highest ascorbic acid $8.85 \mathrm{mg} 100 \mathrm{~g}^{-1}$ followed by $\mathrm{S}_{3} \mathrm{~T}_{3}\left(8.80 \mathrm{mg} 100 \mathrm{~g}^{-1}\right)$ while minimum ascorbic acid was noticed in $\mathrm{S}_{3} \mathrm{~T}_{5}$ i.e. punnets $\left(8.56 \mathrm{mg} 100 \mathrm{~g}^{-1}\right)$. The chief reason for the loss in ascorbic acid was the solubility in water, thermal destruction and enzymatic oxidation during storage (Selmon, 1994). The ascorbic acid content of okra decreased gradually with increase in storage period as reported by Rani, et al. (2015), Sreeramulu, et al. (1983) in leafy vegetables; Jagtap (1986) in fenugreek and spinach; Yadav and Sehgal (1997) in fenugreek; Waskar, et al. (1999) in bottle gourd and Negi and Roy (2004) in amaranthus and fenugreek.

Total yeast and mould count $\left(\log \mathrm{cfu} \mathrm{g}^{-1}\right)$ :-

The effect of packaging materials and storage conditions on total yeast and mould count of okra was found to be non-significant. However, the gradual increase was noticed in total yeast and mould count of okra with the advancement of storage period (Table 3). During storage period, the maximum total yeast and mould count was recorded in the samples stored at ambient temperature $\left(4.85 \log \mathrm{cfu} \mathrm{g}^{-1}\right)$ followed by cool chamber $\left(4.79 \operatorname{log~cfu~g}^{-1}\right)$ and refrigerated storage $\left(4.74 \log \mathrm{cfu} \mathrm{g}^{-1}\right)$. At the end of $15^{\text {th }}$ day storage under refrigerated conditions, the maximum total yeast and mould count was noticed in the treatment $\mathrm{S}_{3} \mathrm{~T}_{5}\left(4.90 \log\right.$ cfu g $\left.\mathrm{g}^{-1}\right)$ followed by $\mathrm{S}_{3} \mathrm{~T}_{1}\left(4.86 \log \mathrm{cfu} \mathrm{g}^{-1}\right)$ whereas the minimum count recorded in $\mathrm{S}_{3} \mathrm{~T} 6\left(4.79 \mathrm{log}_{\mathrm{cfug}}{ }^{-1}\right)$. These results are in agreement with the findings of Ngure, et al. (2009) who reported that okra samples stored in polyethylene recorded low yeast and mould count but very high bacteria counts. This could be because of high moisture content (high water activity level) which gave room for more bacterial growth than yeast and mould count. Babarinda and Fabunmi (2009) who concluded that the high microbial load in these samples could be due to high heat of respiration in the polyethylene bag at the early period of storage.

Table 3:- Effect of packaging materials and storage condition on total yeast and mould count of okra $\left(\log \mathrm{cfu} \mathrm{g}^{-1}\right)$

\begin{tabular}{|c|c|c|c|}
\hline \multirow[t]{2}{*}{ Particulars } & \multicolumn{3}{|c|}{ Total yeast and mould count } \\
\hline & Initial day & Final day count & Storage life \\
\hline \multicolumn{4}{|c|}{ Storage conditions } \\
\hline$S_{1}$ & 3.72 & 4.85 & 3 \\
\hline $\mathrm{S}_{2}$ & 3.72 & 4.79 & 9 \\
\hline $\mathrm{S}_{3}$ & 3.72 & 4.74 & 15 \\
\hline $\mathrm{SE}(\mathrm{m}) \pm$ & - & 0.01 & - \\
\hline CD at $1 \%$ & - & 0.05 & - \\
\hline \multicolumn{4}{|l|}{ Treatment } \\
\hline $\mathrm{T}_{1}$ & 3.72 & 4.82 & 15 \\
\hline$T_{2}$ & 3.72 & 4.81 & 15 \\
\hline $\mathrm{T}_{3}$ & 3.72 & 4.79 & 15 \\
\hline $\mathrm{T}_{4}$ & 3.72 & 4.78 & 15 \\
\hline $\mathrm{T}_{5}$ & 3.72 & 4.85 & 15 \\
\hline $\mathrm{T}_{6}$ & 3.72 & 4.70 & 15 \\
\hline SE $(\mathbf{m}) \pm$ & - & 0.02 & - \\
\hline CD at $1 \%$ & - & 0.06 & - \\
\hline \multicolumn{4}{|l|}{ Interaction } \\
\hline $\mathrm{S}_{1} \mathrm{~T}_{1}$ & 3.72 & 4.79 & 3 \\
\hline $\mathrm{S}_{1} \mathrm{~T}_{2}$ & 3.72 & 4.76 & 3 \\
\hline $\mathrm{S}_{1} \mathrm{~T}_{3}$ & 3.72 & 4.75 & 3 \\
\hline $\mathrm{S}_{1} \mathrm{~T}_{4}$ & 3.72 & 4.74 & 3 \\
\hline $\mathrm{S}_{1} \mathrm{~T}_{5}$ & 3.72 & 4.79 & 3 \\
\hline $\mathrm{S}_{1} \mathrm{~T}_{6}$ & 3.72 & 4.64 & 1 \\
\hline $\mathrm{S}_{2} \mathrm{~T}_{1}$ & 3.72 & 4.81 & 9 \\
\hline $\mathrm{S}_{2} \mathrm{~T}_{2}$ & 3.72 & 4.80 & 9 \\
\hline $\mathrm{S}_{2} \mathrm{~T}_{3}$ & 3.72 & 4.79 & 9 \\
\hline $\mathrm{S}_{2} \mathrm{~T}_{4}$ & 3.72 & 4.77 & 9 \\
\hline
\end{tabular}




\begin{tabular}{|c|c|c|c|}
\hline $\mathrm{S}_{2} \mathrm{~T}_{5}$ & 3.72 & 4.87 & 9 \\
\hline $\mathrm{S}_{2} \mathrm{~T}_{6}$ & 3.72 & 4.67 & 2 \\
\hline $\mathrm{S}_{3} \mathrm{~T}_{1}$ & 3.72 & 4.86 & 15 \\
\hline $\mathrm{S}_{3} \mathrm{~T}_{2}$ & 3.72 & 4.86 & 15 \\
\hline $\mathrm{S}_{3} \mathrm{~T}_{3}$ & 3.72 & 4.84 & 15 \\
\hline $\mathrm{S}_{3} \mathrm{~T}_{4}$ & 3.72 & 4.83 & 15 \\
\hline $\mathrm{S}_{3} \mathrm{~T}_{5}$ & 3.72 & 4.90 & 15 \\
\hline $\mathrm{S}_{3} \mathrm{~T}_{6}$ & 3.72 & 4.79 & 3 \\
\hline $\mathrm{SE}(\mathrm{m}) \pm$ & - & 0.03 & - \\
\hline CD at 1\% & - & NS & - \\
\hline "-' indicate termination & \multicolumn{3}{|c|}{$T_{1^{-}} 150$ gauge polyethylene with $2 \%$ vent } \\
\hline$S_{1}$-Ambient temperature & \multicolumn{3}{|c|}{$\mathrm{T}_{2}-200$ gauge polyethylene with $2 \%$ vent } \\
\hline $\mathrm{S}_{2}$-Zero Energy Cool Chamber & \multicolumn{3}{|c|}{$\mathrm{T}_{3}-300$ gauge polyethylene with $2 \%$ vent } \\
\hline$S_{3}$-Refrigerated storage & \multicolumn{3}{|c|}{$\mathrm{T}_{4}-400$ gauge polyethylene with $2 \%$ vent } \\
\hline & \multicolumn{3}{|c|}{$\mathrm{T}_{5}$ - Punnet } \\
\hline & \multicolumn{3}{|c|}{$\mathrm{T}_{6^{-}}$Control } \\
\hline
\end{tabular}

Organoleptic evaluation:-

The organoleptic score of okra as influenced by different packaging materials and storage conditions revealed that, the decrease in organoleptic score for colour, tenderness, appearance and overall acceptability was rapid at room temperature as compared to zero energy cool chamber and refrigerated storage (Table 4 and 5).

Table 4:- Effect of packaging materials and storage conditions on colour and tenderness of okra during storage

\begin{tabular}{|c|c|c|c|c|c|c|c|c|}
\hline \multirow[b]{3}{*}{ Storage } & \multicolumn{4}{|c|}{ Colour } & \multicolumn{4}{|c|}{ Tenderness } \\
\hline & \multicolumn{4}{|c|}{ Storage period in days } & \multicolumn{4}{|c|}{ Storage period in days } \\
\hline & 0 & 3 & 9 & 15 & 0 & 3 & 9 & 15 \\
\hline $\mathrm{S}_{1}$ & 8.94 & 6.68 & - & 15 & 8.94 & 7.24 & - & - \\
\hline $\mathrm{S}_{2}$ & 8.94 & 7.76 & 6.51 & - & 8.94 & 7.71 & 6.95 & - \\
\hline $\mathrm{S}_{3}$ & 8.94 & 8.48 & 7.60 & - & 8.94 & 8.56 & 7.36 & 6.26 \\
\hline Treatments & & & & 6.49 & & & & \\
\hline $\mathrm{T}_{1}$ & 9.00 & 7.66 & 7.07 & & 9.00 & 7.89 & 7.13 & 6.25 \\
\hline $\mathrm{T}_{2}$ & 9.00 & 7.71 & 7.14 & 6.48 & 9.00 & 7.89 & 7.20 & 6.26 \\
\hline $\mathrm{T}_{3}$ & 9.00 & 7.77 & 7.17 & 6.50 & 9.00 & 7.89 & 7.22 & 6.28 \\
\hline $\mathrm{T}_{4}$ & 9.00 & 7.79 & 7.24 & 6.51 & 9.00 & 7.96 & 7.31 & 6.28 \\
\hline $\mathrm{T}_{5}$ & 9.00 & 7.50 & 6.65 & 6.51 & 9.00 & 7.78 & 6.90 & 6.23 \\
\hline $\mathrm{T}_{6}$ & 8.67 & 7.78 & 0.00 & 6.47 & 8.67 & 7.89 & 0.00 & 0.00 \\
\hline Interaction & & & & 0.00 & & & & \\
\hline $\mathrm{S}_{1} \mathrm{~T}_{1}$ & 9.00 & 6.63 & - & & 9.00 & 7.22 & - & - \\
\hline$S_{1} T_{2}$ & 9.00 & 6.70 & - & - & 9.00 & 7.33 & - & - \\
\hline $\mathrm{S}_{1} \mathrm{~T}_{3}$ & 9.00 & 6.77 & - & - & 9.00 & 7.33 & - & - \\
\hline $\mathrm{S}_{1} \mathrm{~T}_{4}$ & 9.00 & 6.80 & - & - & 9.00 & 7.22 & - & - \\
\hline $\mathrm{S}_{1} \mathrm{~T}_{5}$ & 9.00 & 6.50 & - & - & 9.00 & 7.11 & - & - \\
\hline $\mathrm{S}_{1} \mathrm{~T}_{6}$ & 8.67 & - & - & - & 8.67 & - & - & - \\
\hline $\mathrm{S}_{2} \mathrm{~T}_{1}$ & 9.00 & 7.67 & 6.47 & - & 9.00 & 7.78 & 6.93 & - \\
\hline $\mathrm{S}_{2} \mathrm{~T}_{2}$ & 9.00 & 7.78 & 6.50 & - & 9.00 & 7.67 & 6.96 & - \\
\hline $\mathrm{S}_{2} \mathrm{~T}_{3}$ & 9.00 & 7.89 & 6.57 & - & 9.00 & 7.67 & 7.00 & - \\
\hline $\mathrm{S}_{2} \mathrm{~T}_{4}$ & 9.00 & 7.89 & 6.60 & - & 9.00 & 8.00 & 7.07 & - \\
\hline $\mathrm{S}_{2} \mathrm{~T}_{5}$ & 9.00 & 7.56 & 6.42 & - & 9.00 & 7.44 & 6.80 & - \\
\hline $\mathrm{S}_{2} \mathrm{~T}_{6}$ & 8.67 & - & - & - & 8.67 & - & - & - \\
\hline $\mathrm{S}_{3} \mathrm{~T}_{1}$ & 9.00 & 8.67 & 7.67 & - & 9.00 & 8.67 & 7.33 & 6.25 \\
\hline $\mathrm{S}_{3} \mathrm{~T}_{2}$ & 9.00 & 8.67 & 7.78 & 6.48 & 9.00 & 8.67 & 7.44 & 6.26 \\
\hline $\mathrm{S}_{3} \mathrm{~T}_{3}$ & 9.00 & 8.67 & 7.78 & 6.50 & 9.00 & 8.67 & 7.44 & 6.28 \\
\hline $\mathrm{S}_{3} \mathrm{~T}_{4}$ & 9.00 & 8.67 & 7.89 & 6.51 & 9.00 & 8.67 & 7.56 & 6.28 \\
\hline $\mathrm{S}_{3} \mathrm{~T}_{5}$ & 9.00 & 8.44 & 6.89 & 6.51 & 9.00 & 8.78 & 7.00 & 6.23 \\
\hline
\end{tabular}




\begin{tabular}{|c|c|c|c|c|c|c|c|c|}
\hline $\mathrm{S}_{3} \mathrm{~T}_{6}$ & 8.67 & 7.78 & - & 6.47 & 8.67 & 7.89 & - & - \\
\hline & & & & - & & & & \\
\hline \multicolumn{4}{|c|}{ "- indicate termination } & \multicolumn{5}{|c|}{$\mathrm{T}_{1^{-}}-150$ gauge polyethylene with $2 \%$ vent } \\
\hline \multicolumn{4}{|c|}{$\mathrm{S}_{1}$-Ambient temperature } & \multicolumn{5}{|c|}{$\mathrm{T}_{2}-200$ gauge polyethylene with $2 \%$ vent } \\
\hline \multicolumn{4}{|c|}{$\mathrm{S}_{2}$-Zero Energy Cool Chamber } & \multicolumn{5}{|c|}{$\mathrm{T}_{3}-300$ gauge polyethylene with $2 \%$ vent } \\
\hline \multirow{3}{*}{\multicolumn{4}{|c|}{$S_{3}-$ Refrigerated storage }} & \multicolumn{5}{|c|}{$\mathrm{T}_{4}-400$ gauge polyethylene with $2 \%$ vent } \\
\hline & & & & \multicolumn{5}{|c|}{$\mathrm{T}_{5}$ - Punnet } \\
\hline & & & & \multicolumn{5}{|c|}{$\mathrm{T}_{6^{-}}$Control } \\
\hline
\end{tabular}

Table 5:- Effect of packaging materials and storage conditions on appearance and overall acceptability of okra during storage

\begin{tabular}{|c|c|c|c|c|c|c|c|c|}
\hline & \multicolumn{4}{|c|}{ Appearance } & \multicolumn{4}{|c|}{ Overall acceptability } \\
\hline & \multicolumn{4}{|c|}{ Storage period in days } & \multicolumn{4}{|c|}{ Storage period in days } \\
\hline Storage & 0 & 3 & 9 & 15 & 0 & 3 & 9 & 15 \\
\hline $\mathrm{S}_{1}$ & 8.94 & 6.91 & - & - & 8.67 & 7.11 & - & - \\
\hline $\mathrm{S}_{2}$ & 8.94 & 7.78 & 6.75 & - & 8.67 & 7.62 & 6.17 & - \\
\hline $\mathrm{S}_{3}$ & 8.94 & 8.77 & 7.49 & 6.33 & 8.67 & 8.06 & 7.58 & 6.18 \\
\hline \multicolumn{9}{|c|}{ Treatments } \\
\hline $\mathrm{T}_{1}$ & 9.00 & 7.74 & 7.03 & 6.31 & 8.67 & 7.63 & 6.80 & 6.17 \\
\hline $\mathrm{T}_{2}$ & 9.00 & 7.79 & 7.15 & 6.33 & 8.67 & 7.70 & 6.87 & 6.18 \\
\hline $\mathrm{T}_{3}$ & 9.00 & 7.90 & 7.23 & 6.34 & 8.67 & 7.78 & 6.94 & 6.18 \\
\hline $\mathrm{T}_{4}$ & 9.00 & 7.98 & 7.34 & 6.35 & 8.67 & 7.81 & 7.05 & 6.20 \\
\hline$T_{5}$ & 9.00 & 7.78 & 6.83 & 6.30 & 8.67 & 7.52 & 6.71 & 6.17 \\
\hline $\mathrm{T}_{6}$ & 8.67 & 8.50 & 0.00 & 0.00 & 8.67 & 6.67 & 0.00 & 0.00 \\
\hline \multicolumn{9}{|l|}{ Interaction } \\
\hline $\mathrm{S}_{1} \mathrm{~T}_{1}$ & 9.00 & 6.90 & - & - & 8.67 & 7.00 & - & - \\
\hline$S_{1} T_{2}$ & 9.00 & 6.92 & - & - & 8.67 & 7.11 & - & - \\
\hline $\mathrm{S}_{1} \mathrm{~T}_{3}$ & 9.00 & 6.92 & - & - & 8.67 & 7.22 & - & - \\
\hline $\mathrm{S}_{1} \mathrm{~T}_{4}$ & 9.00 & 6.93 & - & - & 8.67 & 7.33 & - & - \\
\hline $\mathrm{S}_{1} \mathrm{~T}_{5}$ & 9.00 & 6.88 & - & - & 8.67 & 6.89 & - & - \\
\hline $\mathrm{S}_{1} \mathrm{~T}_{6}$ & 8.67 & - & - & - & 8.67 & - & - & - \\
\hline $\mathrm{S}_{2} \mathrm{~T}_{1}$ & 9.00 & 7.67 & 6.73 & - & 8.67 & 7.56 & 6.15 & - \\
\hline$S_{2} T_{2}$ & 9.00 & 7.78 & 6.75 & - & 8.67 & 7.67 & 6.19 & - \\
\hline $\mathrm{S}_{2} \mathrm{~T}_{3}$ & 9.00 & 7.89 & 6.78 & - & 8.67 & 7.78 & 6.20 & - \\
\hline $\mathrm{S}_{2} \mathrm{~T}_{4}$ & 9.00 & 8.00 & 6.80 & - & 8.67 & 7.78 & 6.21 & - \\
\hline $\mathrm{S}_{2} \mathrm{~T}_{5}$ & 9.00 & 7.56 & 6.67 & - & 8.67 & 7.33 & 6.09 & - \\
\hline $\mathrm{S}_{2} \mathrm{~T}_{6}$ & 8.67 & - & - & - & 8.67 & - & - & - \\
\hline $\mathrm{S}_{3} \mathrm{~T}_{1}$ & 9.00 & 8.67 & 7.33 & 6.31 & 8.67 & 8.33 & 7.44 & 6.17 \\
\hline $\mathrm{S}_{3} \mathrm{~T}_{2}$ & 9.00 & 8.67 & 7.56 & 6.33 & 8.67 & 8.33 & 7.56 & 6.18 \\
\hline $\mathrm{S}_{3} \mathrm{~T}_{3}$ & 9.00 & 8.89 & 7.67 & 6.34 & 8.67 & 8.33 & 7.67 & 6.18 \\
\hline $\mathrm{S}_{3} \mathrm{~T}_{4}$ & 9.00 & 9.00 & 7.89 & 6.35 & 8.67 & 8.33 & 7.89 & 6.20 \\
\hline $\mathrm{S}_{3} \mathrm{~T}_{5}$ & 9.00 & 8.89 & 7.00 & 6.30 & 8.67 & 8.33 & 7.33 & 6.17 \\
\hline $\mathrm{S}_{3} \mathrm{~T}_{6}$ & 8.67 & 8.50 & - & - & 8.67 & 6.67 & - & - \\
\hline \multicolumn{4}{|c|}{ '-" indicate termination } & \multicolumn{5}{|c|}{$\mathrm{T}_{1}-150$ gauge polyethylene with $2 \%$ vent } \\
\hline \multicolumn{4}{|c|}{$S_{1}-$ Ambient temperature } & \multicolumn{5}{|c|}{$\mathrm{T}_{2}-200$ gauge polyethylene with $2 \%$ vent } \\
\hline \multicolumn{4}{|c|}{$\mathrm{S}_{2}$-Zero Energy Cool Chamber } & \multicolumn{5}{|c|}{$\mathrm{T}_{3}-300$ gauge polyethylene with $2 \%$ vent } \\
\hline \multirow{3}{*}{\multicolumn{4}{|c|}{$S_{3}$-Refrigerated storage }} & \multicolumn{5}{|c|}{$\mathrm{T}_{4}-400$ gauge polyethylene with $2 \%$ vent } \\
\hline & & & & \multicolumn{5}{|c|}{$T_{5}$ - Punnet } \\
\hline & & & & \multicolumn{5}{|c|}{$\mathrm{T}_{6^{-}}$Control } \\
\hline
\end{tabular}

The okra fruits packed in 400 gauge polyethylene with $2 \%$ vents and stored in refrigerated storage recorded the highest score for colour (6.51), tenderness, (6.28) appearance (6.35) and overall acceptability (6.20) at the end of $15^{\text {th }}$ day storage followed by $S_{3} T_{3}$ i. e. 300 gauge polyethylene with $2 \%$ vents. The rapid loss of texture of fruits and vegetables attributed to their exposure to atmospheric gases with resultant fading out of their greenish colour. The 
physical damage increases respiration and ethylene production with associated increases in the rate of other reactions responsible for biochemical changes in colour, texture and nutritional quality. The effect of carbon dioxide and chlorophyll retention was attributed to inhibition of ethylene production and low water activity (Martinez and Whitaker, 1995). The results of present study are in close conformity with the results reported by Jagtap (1986) in spinach and Ngure et al. (2009) in okra. Refrigerated storage found to be effective in maintaining the colour and appearance, texture, quality throughout the storage period and maximum overall acceptability might be due to low temperature during storage which led to reduced minimum moisture and physiological loss in weight. The present findings are in agreement with the results reported by Kim et al. (2004) in salad savoy and Komolafe and Idah (2008) in okra.

From the present study it can be concluded that, the shelf life of okra was found to be increased up to three days at room temperature, nine days at zero energy cool chamber and fifteen days at refrigerated storage when the samples packed in 400 gauge polyethylene bags with $2 \%$ vents which helps in better retention of quality parameters. There was a reduction in microbial count under refrigerated samples as compared with the ambient temperature. A gradual decrease was observed in organoleptic score for colour, tenderness, appearance and overall acceptability during storage of okra.

\section{References:-}

1. Abe, K. and Watada, A. E. (1991). Ethylene absorbent to maintain quality of lightly processed fruits and vegetables. J. Food Sci., 56:1589-1592.

2. Amerine, M. S , Pangbore, R M and Rossler, E A. (1965). Principles of Sensory Evaluation of Food. Academic Press, New York.

3. Anonymous, (2011). Microbial test limits, standard procedures. From www.bis.org.in prescribed by USFDA (BAM) IS 5401: 2002, 5402:200, Chapter No.: 1 to 9.

4. Anonymous, (2014). Indian Horticulture Database. National Horticulture Board, Ministry of Agriculture, Govt. of India, pp: 146-148.

5. AOAC, (1990). Official Method of Analysis. $15^{\text {th }}$ ed. Association of Official Analytical Chemist, Washington, DC, USA.

6. Babarinde, G. O. and Fabunmi O. A. (2009). Effects of packaging materials and storage temperature on quality of fresh okra (Abelmoschus esculentus) fruit. Agriculture Tropical and Subtropical. Vol. 42 (4):151-156.

7. Gopalkrishnan, T. R. (2007). Vegetable Crops. Horticulture Science Series Vol. 4., New India Publishing Agency, Pitum Pura, New Delhi- 410088.

8. Jagtap, B. (1986). Effect of foliar spray of growth substance on the growth yield and shelf life of leafy vegetables viz., Fenugreek and Spinach. A M. Sc. (Agri.) thesis submitted to Mahatma Phule Krishi Vidyapeeth, Rahuri (M. S.).

9. Jain A., Sharma, S. R., Mittal, T. C and Gupta, S. K. (2014). Storage studies of okra under ambient storage conditions. Intl. J. of Innovative Res. and Technol., 1 (4):1-8.

10. Kim, J. G., Luo, Y. and Gross, K. C. (2004). Effect of package film on the quality of fresh cut salad savoy. Post-harvest Biol. Tech., 32:99-107.

11. Komolafe, G. O. and Idah, P. A. (2008). Effect of temperature and time on moisture content, texture and texture of okra. Nig. Food Journal, 26(2):106-113.

12. Koraddi, V. (2005). Methods of storage of vegetables in the refrigerator at household level. M. Sc. thesis submitted to University of Agricultural Sciences, Dharwad (Karnataka).

13. Leonard, E. A. (1987). Packaging, Specification, Purchasing and Quality Control. Marcel Dekker, New York, pp: 4.

14. Martinez, M. and Whitaker, J. R. (1995). The biochemistry and control of enzyme browning. Trends Food Sci. Technol. 6:195-200.

15. Negi, P. S. and Roy, S. K. (2004). Changes in $\beta$-carotene and ascorbic acid content of fresh amaranthus and fenugreek leaves during storage by low cost technique. Plant Food for Human Nutrition, 58:225-230.

16. Ngure, J. W., Joseph, N., Aguyoh, G. and Goaquiong, L. (2009). Interactive effects of packaging and storage temperatures on the shelf life of okra. ARPN J. Agric. Bio. Sci., 4(3):43-52.

17. Panse, V. G. and Sukhatme, P. V. (1985). Statistical Methods of Agricultural Workers, ICAR, New Delhi. pp: 143-147.

18. Rai, D. R., Narsaiah, K., Bharati, D. K., Reddy, P. M. K. and Brar, S. S. (2009). Modified atmosphere packaging of minimal processing and storage of shredded cabbage. J. Food Sci. Technol., 46(5):436-439. 
19. Rani, M. Singh, J. and Kumar, D. (2015). Effect of different packaging material on chlorophyll and ascorbic acid content of the okra. South Asian J. Food Technol. Environ. 1(1):86-88.

20. Reddy, J. B., Bharati, P, Naik, K. R., Chimmad, B. V., Itagi, S. K. and Hasalkar, S. (2013). Effect of packaging materials on shelf life of minimally processed rajgira leaves (Amaranthus paniculatus). Karnataka J. Agric. Sci., 26(2):285-287.

21. Roy, S. K. and Khurdiya, D. S. (1986). Studies on evaporatively cooled zero energy input cool chamber for the storage of horticultural produce. Indian Food Packer, 40:26-31.

22. Santi, R. , Bhowmilk, S. R. and Jung, C. P. (1992). Shelf life of mature green tomatoes stored in controlled atmosphere and high humidity. J. Food Sci., 57:948-951.

23. Selmon, I. D. (1994). Vitamin retention during blanching of vegetables. Food Chemistry, 49:137-147.

24. Sreeramulu, N. , Banyikwa, F. F. and Shrivastava, V. 1983.Loss of ascorbic acid due to wilting in some green leafy vegetables. Pl. Foods. 5(4):215-219.

25. Waskar, D. P, Yadav, B. B. and Garande, V. K. (1999). Influence of various packaging materials on storage behavior of bottle gourd under different storage conditions. Indian J. Agric. Res. 33(4): 287-292.

26. Wills, R. B. H., Mcglasson, W. B., Graham, D., Lee, T. H. and Hall, E . G. (1989). Post-harvest: An introduction to the physiology and handling of fruits and vegetables. South China Printing Company Limited, $3^{\text {rd }}$ edn., pp: $170-190$.

27. Yadav, S. K. and Sehgal, S. (1997). Effect of home processing and storage on ascorbic acid and $\beta$ - carotene content of Bathua (Chenopodium album) and fenugreek (Trigonella foenumgraecum) leaves. Plant Foods for Human Nutrition 50: 239-247. 